\title{
Vers une projection des familles selon leurs caractéristiques principales
}

\section{TOWARDS A PROJECTION OF FAMILIES ACCORDING TO THEIR PRINCIPAL CHARACTERISTICS HACIA UNA PROYECCIÓN DE LAS FAMILIAS SEGUN SUS CARACTERISTICAS PRINCIPALES}

\section{Jacques Ledent}

Volume 24, numéro 1, printemps 1995

Perspective de population

URI : https://id.erudit.org/iderudit/010180ar

DOI : https://doi.org/10.7202/010180ar

Aller au sommaire du numéro

Éditeur(s)

Association des démographes du Québec

ISSN

0380-1721 (imprimé)

1705-1495 (numérique)

Découvrir la revue

Citer cet article

Ledent, J. (1995). Vers une projection des familles selon leurs caractéristiques principales. Cahiers québécois de démographie, 24(1), 3-33.

https://doi.org/10.7202/010180ar
Résumé de l'article

Cet article a pour objet de décrire les grandes lignes d'une méthodologie en cours d'élaboration visant à projeter les familles (ou groupes d'individus ayant entre eux des liens conjugaux ou de sang) selon leurs caractéristiques principales, à savoir le genre de famille mais aussi le nombre et l'âge des enfants présents. Cette méthodologie s'appuie pour l'essentiel sur un modèle de type multi-dimensionnel se présentant comme une extension de la traditionnelle méthode des composantes à la prise en compte du statut conjugal et de la parité des femmes. En bout de course, moyennant un élargissement approprié de la variable de statut conjugal et un prolongement axé sur le cas particulier des familles recomposées, elle autorise un passage immédiat des effectifs de la population féminine selon le statut démographique (issu du croisement selon l'état conjugal et le nombre d'enfants présents) aux effectifs des familles selon leurs caractéristiques principales.
Tous droits réservés @ Association des démographes du Québec, 1995

Ce document est protégé par la loi sur le droit d'auteur. L'utilisation des services d'Érudit (y compris la reproduction) est assujettie à sa politique d'utilisation que vous pouvez consulter en ligne.

https://apropos.erudit.org/fr/usagers/politique-dutilisation/ 
Cahiers québécois de démographie

Vol. 24, no 1, printemps 1995, p. 3-33.

\title{
Vers une projection des familles selon leurs caractéristiques principales
}

\author{
Jacques LEDENT *
}

Depuis le début des années 1960, le monde occidental est en proie à une profonde mutation démographique attribuable à des modifications de comportement fortement marquées. Ainsi, pour ne citer que les tendances les plus significatives, se sont enchaînées une baisse substantielle de la fécondité (principalement dans les années 1960), une baisse considérable de la nuptialité accompagnée d'une hausse importante de la divortialité (fin des années 1960, années 1970) et enfin un fort accroissement de la fécondité hors mariage (années 1980), encore que cet ordre de succession effectivement observé en Amérique du Nord ait ailleurs été assorti de chevauchements, plus ou moins accusés selon les pays. Une telle accumulation de tendances s'est naturellement traduite par une transformation appréciable de la structure des familles, laquelle, malgré l'absence d'une nouvelle tendance forte, se poursuit aujourd'hui du fait de l'action retardée des tendances passées, en particulier en matière de fécondité.

Parallèlement à ces modifications de comportement démographique se sont également produites - indépendamment ou non - des modifications de comportement économique qui, de leur côté, ont contribué à modifier la structure des ménages. Mais cet autre type de modifications de comportement, fort intéressant au demeurant, n'entre pas dans le cadre de cet article axé sur les familles, c'est-à-dire les groupements d'individus en unités domestiques sur la base de liens de nature démographique, c'est-à-dire de liens conjugaux et (ou) de liens du sang. C'est donc à dessein que nous y laissons dans l'ombre les ménages, c'est-à-dire les groupements de familles en unités de logement sur la base de liens de nature économique.

* INRS-Urbanisation, Université du Guébec, Montréal, Québec. 
L'idée incontournable que la structure des familles va continuer à se modifier à l'avenir suggère évidemment au démographe une certaine obligation d'anticiper la nature et l'importance des changements susceptibles de se produire de manière à éclairer les ajustements à apporter aux politiques gouvernementales ayant un rapport étroit avec la structure des familles, notamment les politiques d'allocations d'aide aux jeunes enfants (allocations familiales) et aux personnes âgées (pensions). Une telle obligation pose même au démographe un défi de taille car, afin de projeter l'évolution des familles sur la base d'hypothèses appropriées au niveau des événements démographiques responsables de cette évolution, il ne dispose d'aucun outil suffisamment établi, tel que peut l'être par exemple la méthode classique des composantes servant à projeter la population sur la base d'hypothèses adéquates de fécondité, de mortalité et de migration. La méthode des taux de personnes repères, couramment utilisée par les bureaux statistiques nationaux pour préparer des projections de familles et de ménages, se révèle en effet impuissante à anticiper les changements attendus. Si elle est malléable au point de pouvoir sous-tendre une projection des familles suivant une nomenclature détaillée selon le genre, la taille et la composition, cette méthode se prête très mal, comme nous le verrons plus loin, à la prise en considération explicite des événements démographiques responsables de la formation et de la dissolution des familles.

La disponibilité d'un outil approprié pour projeter l'évolution des familles ne serait-elle qu'une chimère? Pour notre part, les perspectives ouvertes par l'émergence de la dêmographie mathématique multidimensionnelle (voir par exemple Land et Rogers, 1982) nous incitent à croire qu'il n'en est rien. Suivant en cela la voie tracée par plusieurs chercheurs, notamment Bongaarts (1987), nous nous sommes engagé il y a plusieurs années à recourir aux méthodes et techniques de cette nouvelle branche de la démographie afin de mettre au point l'outil nécessaire (Ledent, Péron et Morissette, 1987). Au fil des années, notre démarche s'est peaufinee (Ledent, 1992) et, tout récemment encore, elle s'est enrichie de développements nouveaux qui permettent d'envisager la réalisation d'une application concrète dans un avenir rapproché (Ledent, 1995).

Dans cet article, notre propos n'est pas de présenter en détails la formulation du modèle de projection auquel nous avons abouti - une telle présentation reste à faire - mais 
plutôt de décrire les grandes lignes de la méthodologie, à la fois cohérente et cohésive, qui la sous-tend. La description envisagée s'inscrit dans une perspective didactique visant à mettre à la portée d'un auditoire aussi large que possible cette méthodologie qui, en raison de l'utilisation abondante qu'elle fait de la notation matricielle, est susceptible de rebuter nombre de démographes potentiellement intéressés.

\section{CONSIDÉRATIONS PRÉLIMINAIRES}

Avant d'entamer la description mème de la méthodologie, voyons tout d'abord pourquoi et comment elle nous parait capable de remédier aux insuffisances de la méthode des taux de personnes repères.

\section{De la méthode des taux de personnes repères à une méthodologie de type multidimensionnel}

Traditionnellement, la projection des familles et des ménages s'effectue à l'aide de la méthode des taux de chefs, aujourd'hui rebaptisée méthode des taux de personnes repères. Telle qu'elle a été définie originellement il y a plus d'un demisiècle (USNRC, 1938), cette méthode, d'ailleurs utilisée sans modifications jusqu'à il y a peu, consiste à appliquer à des effectifs de population selon le sexe et l'âge projetés au préalable des proportions - dites taux de personnes repères fixées de manière exogène, généralement sur la base d'extrapolations d'un petit nombre d'observations antérieures. Il s'agit donc d'une méthode simple et transparente qui, si elle utilise peu d'intrants, ne livre en contrepartie que peu d'extrants (Bongaarts, 1983).

Dans un passé récent, cependant, la méthode des taux de personnes repères a donné lieu à nombre de raffinements destinés à fournir aux utilisateurs des extrants plus développés (voir Kono, 1987; Larrivée, 1990). Si le principe de la méthode originelle demeure, le nombre d'intrants (et par suite d'extrants) a augmenté, rendant l'application de la méthode de plus en plus exigeante mais aussi de moins en moins fiable à mesure que ce nombre augmente.

En fait, la méthode des taux de personnes repères souffre d'un défaut rédhibitoire lié à l'emploi qu'elle fait d'un ensemble de proportions et ratios dont les valeurs, issues de simples extrapolations temporelles, n'ont aucun contenu explicatif. De 
manière spécifique, elle occulte le lien existant entre les paramètres utilisés comme intrants et les événements démographiques justifiant les valeurs prises par ces paramètres, au niveau tant de sa nature que de sa dimension temporelle. D'une part, la formulation de la méthode ne repose sur aucune prise en considération explicite des événements clés de la formation et de la dissolution des familles; d'autre part, elle fait appel à des intrants de nature cumulative puisqu'ils se rapportent à des changements démographiques intervenus non pas sur le pas de la projection mais sur la durée de vie des individus.

La méthode des taux de personnes repères, tant dans sa version originelle que sous la forme de ses raffinements les plus récents, est donc une méthode foncièrement mécanique et statique. En conséquence, elle ne peut sous-tendre une projection satisfaisante des familles selon leurs caractéristiques principales, laquelle se doit i) de lier de manière explicite la structure des familles aux événements démographiques qui l'expliquent et ii) de mettre en œuvre cette liaison à chaque pas de la projection envisagée.

A priori, une telle projection doit pouvoir être réalisée au moyen d'une méthodologie empruntant à la démographie mathématique multidimensionnelle. En effet, le propre de cette nouvelle branche de la démographie est de suivre l'évolution d'une cohorte de personnes nées en une même année à mesure que celle-ci diminue en taille, non seulement sur le plan de son importance numérique mais aussi sur le plan de sa distribution parmi divers états associés à une variable de statut démographique. Ainsi a-t-il été possible d'élargir la méthode des composantes à la prise en compte de la région de résidence (Rogers, 1968, 1975), du statut d'activité (Hoem et Fong, 1976; Willekens, 1980) ou encore de l'état matrimonial (Schoen et Land, 1979; Willekens et al., 1982). De la même manière, il devrait être possible de l'élargir à la prise en compte d'états issus de croisements pertinents d'un petit nombre de variables de statut démographique et ce - il s'agit là d'un point essentiel - de façon à ce qu'en bout de course ces états autorisent un regroupement quasi automatique des invidus en familles.

\section{Vers un modèle de type multidimensionnel}

Entamant maintenant notre quête pour un modèle de type multidimensionnel approprié, identifions tout d'abord les variables de statut démographique à introduire dans la méthode classique des composantes de manière à autoriser le 
regroupement quasi automatique des individus en familles. Dès le départ, deux variables s'imposent : 1) l'état conjugal de la ou des personnes principales de la famille et 2) le nombre et idéalement l'âge des enfants vivant auprès d'elle(s) ${ }^{1}$.

Cependant, si novatrices soient-elles, les méthodes et techniques usuelles de la démographie multidimensionnelle ne permettent pas de suivre de manière simultanée l'êvolution des caractéristiques relatives à deux ou plusieurs membres d'une famille, que ceux-ci soient les deux conjoints ou encore un parent et son ou ses enfants.

Cette limite, quoique fâcheuse, n'en est pas pour autant fatale. D'une part, au regard du premier problème posé par la présence de deux conjoints, la solution consiste à choisir dans chaque famille une personne repère qui, étant donné le lien privilégié existant entre mère et enfants, est de préférence une femme. Pour le cas où la personne repère est nécessairement un homme (cas d'une famille monoparentale à parent masculin), elle commande alors de suivre ce dernier non pas directement (c'est-à-dire à l'aide d'un modèle de projection englobant les hommes), mais indirectement (par le truchement de valeurs additionnelles au niveau de l'état conjugal), au travers de la mère des enfants, que celle-ci soit décédée ou qu'elle vive seule ou avec un nouveau conjoint. Cela a d'ailleurs pour effet de circonscrire le modèle multidimensionnel à construire à la seule population féminine. Par ailleurs, on peut contourner le second problème relatif au suivi simultané d'un parent, donc le plus souvent de la mère, et de ses enfants en faisant tourner le modèle non pas une seule fois mais plusieurs, en fait autant de fois qu'il existe d'années pour lesquelles une projection des effectifs de la population féminine selon le nombre et l'âge des enfants est souhaitée. Il n'en reste pas moins que ces palliatifs ne permettent pas d'éliminer toutes les difficultês car, comme nous le verrons plus loin, ils laissent en suspens une "zone grise", à savoir la détermination du nombre et de l'âge des enfants dans les familles recomposées, qui doit alors être réglée dans le cadre d'un prolongement ad hoc du modèle.

En résumé, afin de projeter les familles selon leurs caractéristiques principales, il suffit de construire un modèle de projection des femmes selon leur état conjugal ainsi que le

L'âge des enfants importe dans la mesure où les allocations familiales versées par les gouvernements sont modulées selon l'âge des enfants. Ainsi, au Québec, l'allocation d'aide versée aux enfants de moins de 18 ans est majorée dans le cas des jeunes enfants (ceux qui ont moins de 6 ans). 
nombre et l'âge des enfants vivant auprès d'elles. Toutefois, afin d'y parvenir intégralement, il y a lieu de compléter ce modèle en prévoyant 1) une extension de la notion d'état conjugal nécessaire à la prise en considération des familles monoparentales à parent masculin et 2) un prolongement du modèle destinè au traitement particulier requis dans le cas des familles recomposées.

Dans le reste de cet article, nous nous pencherons avant tout sur le "cœur" de notre méthodologie, c'est-à-dire le modèle de projection de la population féminine selon les deux caractéristiques retenues. Ce modèle étant nécessairement complexe, nous nous garderons bien de l'exposer d'un seul trait. Aussi commencerons-nous par examiner une version du modèle réduite à la seule prise en compte du statut conjugal mais, selon nous, expressive au point de révéler les principes généraux sous-tendant une version achevée. Ce n'est que suite à cet examen que nous aborderons une version plus avancée qui introduit la procédure servant à déterminer le nombre et l'âge des enfants effectivement présents. Il sera alors temps d'explorer la manière de passer des effectifs de la population féminine à ceux des familles.

Prêcisons, avant d'entrer dans le vif du sujet, qu'en raison de notre perspective avant tout didactique, ce texte pourrait laisser le lecteur averti sur sa faim. Seule la dernière section, relative au passage des effectifs de population féminine aux nombres de familles, introduit des matériaux vëritablement novateurs. En effet, le modèle utilisé pour projeter les effectifs de la population fémimine (deuxième et troisième sections) s'inspire très largement du modèle originel de Bongaarts (1987), encore que celui-ci soit passablement clarifié (exposition en deux étapes logiques se présentant comme deux extensions successives de la méthode classique des composantes), amendé (élimination de la réalisation séparée des changements d'état conjugal et des changements de parité) et précisé (au moyen d'équations pertinentes).

\section{L'EXTENSION DE LA MÉTHODE DES COMPOSANTES À LA PRISE EN COMPTE DU STATUT CONJUGAL}

Comme nous l'avons annoncé, nous nous proposons dans cette section de montrer comment introduire l'état conjugal dans la méthode des composantes. Précisons que le terme "statut conjugal" est utilisé ici de préférence au terme "êtat 
matrimonial". Contrairement à ce dernier, le premier n'a pas de connotation légale et donc recouvre les situations de couple fondées sur la cohabitation au même titre que celles fondées sur le mariage. Ajoutons que, puisque l'objet de cet article est avant tout d'ordre méthodologique, il n'y a pas lieu ici de présenter une typologie d'états conjugaux et de mettre en évidence les passages effectivement possibles entre les divers états de cette typologie. La nature même de la typologie n'a en effet aucun impact sur la formulation du modèle.

\section{La méthode classique des composantes}

Rappelons que la méthode classique des composantes consiste à déduire l'effectif $\left(\mathrm{K}_{\mathrm{x}+1}\right)$ de toute cohorte d'individus nés en une même année de l'effectif correspondant $\left({ }_{i} \mathrm{~K}_{\mathrm{x}}\right)$ une année plus tôt en soustrayant les décès $\left(D_{x}\right)$ et, dans le cas d'une population ouverte, en prenant en compte les entrées $\left(\mathrm{I}_{x}\right)$ et les sorties $\left(E_{x}\right)$ dues à la migration. De manière plus précise, elle s'appuie sur l'équation de mouvements suivante :

$$
{ }_{\mathrm{f}} \mathrm{K}_{\mathrm{x}+1}={ }_{\mathrm{i}} \mathrm{K}_{\mathrm{x}}-\mathrm{D}_{\mathrm{x}}-\mathrm{E}_{\mathrm{x}}+\mathrm{I}_{\mathrm{x}}
$$

où les souscrits i ( $=$ initial) et $\mathrm{f}$ (= final) placés à gauche du symbole $K$ reflètent le dêbut et la fin de la période annuelle considérée, et le souscrit $x$ placé à droite de chaque symbole l'âge initial (en début de période) des membres de la cohorte.

Étant donné leurs natures démographiques respectives, les entrées dues à la migration sont incorporées sous forme de nombres absolus tandis que les sorties dues tant à la mortalité qu'à la migration le sont sous forme de taux annuels :

$$
\mathrm{d}_{\mathrm{x}}=\mathrm{D}_{\mathrm{x}} / \hat{\mathrm{K}}_{\mathrm{x}}
$$

et

$$
\mathrm{e}_{\mathrm{x}}=\mathrm{E}_{\mathrm{x}} / \hat{\mathrm{K}}_{\mathrm{x}}
$$

où $\widehat{K}_{x}$ est la population soumise au risque de mortalité et de migration.

En supposant que cette population soumise au risque est la moyenne arithmétique des effectifs initial $\left({ }_{i} K_{x}\right)$ et final $\left({ }_{f} K_{x+1}\right)$, on peut aisément combiner les équations de taux (2) et (3) avec l'équation de mouvements (1). Il s'ensuit une équation où l'effectif final $\left(\mathrm{K}_{\mathrm{f}+1}\right)$ apparait comme une simple fonction linéaire de l'effectif initial $\left(\mathrm{K}_{\mathrm{i}}\right)$ :

$$
{ }_{\mathrm{f}} \mathrm{K}_{\mathrm{x}+1}=\mathrm{S}_{\mathrm{x} i} \mathrm{~K}_{\mathrm{x}}+\mathrm{S}_{\mathrm{x}}^{\prime} \mathrm{I}_{\mathrm{x}}
$$


Le premier terme de cette expression s'interprète comme le nombre de survivants parmi les membres du groupe initial ${ }_{i} K_{x}$ - le facteur

$$
\mathrm{s}_{\mathrm{x}}=\left(1-0,5 \mathrm{~m}_{\mathrm{x}}\right) /\left(1+0,5 \mathrm{~m}_{\mathrm{x}}\right)
$$

où $\mathrm{m}_{\mathrm{x}}$ est le taux global de sortie :

$$
\mathrm{m}_{\mathrm{x}}=\mathrm{d}_{\mathrm{x}}+\mathrm{e}_{\mathrm{x}}
$$

exprimant la proportion de survie correspondante - et le second comme le nombre de survivants parmi les immigrants $\mathrm{I}_{\mathrm{x}}$ entrés en cours de période - le facteur

$$
s_{x}^{\prime}=1 /\left(1+0,5 m_{x}\right)
$$

exprimant la proportion de survie (entre l'arrivée et la fin de la période) spécifique à ceux-ci.

Naturellement, dans le cas du premier groupe d'âge $(x=-1)$, il faut modifier l'équation de mouvements (1) afin de tenir compte de la natalité. De fait, l'introduction des naissances par le biais de taux annuels de fécondité

$$
\mathrm{f}_{\mathrm{x}}=\mathrm{N}_{\mathrm{x}} / \hat{\mathrm{k}}_{\mathrm{x}}
$$

conduit, en lieu et place de (4), à :

$$
\mathrm{K}_{0}=\mathrm{S}_{-1}\left(\mathrm{~N}_{.}+\mathrm{I}_{-1}\right)
$$

où $s_{-1}^{\prime}$ est la proportion de survie liée à $m_{-1}$ par la formule générale liant $s_{x}^{\prime}$ à $m_{x}$; cette proportion se trouve donc appliquée d'une part au nombre total de nouveau-nés

$$
\mathrm{N}_{\mathrm{s}}=\sum_{\mathrm{x}} \mathrm{f}_{\mathrm{x}}\left(\left(1+\mathrm{S}_{\mathrm{x}}\right)_{\mathrm{i}} \mathrm{K}_{\mathrm{x}}+\mathrm{I}_{\mathrm{x}}\right) / 2
$$

et d'autre part au nombre d'immigrants $I_{-1}$ relatif au premier groupe d'âge.

\section{Prise en compte du statut conjugal}

L'extension de la méthode des composantes à la prise en compte du statut conjugal ne pose guère de problèmes de principe. Pour la réaliser, il suffit de désagréger chaque cohorte en autant de groupes qu'il existe d'états conjugaux et d'etablir, pour chacun de ces groupes, d'une part les équations de mouvements correspondantes, et d'autre part les équations définissant les taux démographiques relatifs aux mouvements pertinents.

$\mathrm{Au}$ niveau de chacun des $\mathrm{M}$ états conjugaux considérés, les mouvements ne sont plus, comme dans la méthode classique des composantes, limités à des sorties par mortalité et par émigration et à des entrées par immigration. Ils incluent 
maintenant des sorties de même que des entrées dues à des changements de statut conjugal. Dans ces conditions, on peut reprendre l'équation (1), applicable dans le cas où $x \geq 0$, en $y$ affectant chaque terme du superscrit $m$ représentatif du statut conjugal mais aussi en prenant soin d'y faire figurer les sorties vers et les entrées en provenance des autres états conjugaux. Pour ce faire, introduisons le nombre $\left(\mathrm{O}_{\mathrm{x}}^{\mathrm{mn}}\right)$ de personnes qui, à un moment donné au cours de la période considérée, voient leur statut conjugal passer de la valeur $m$ à la valeur $\mathrm{n}$. Il s'ensuit que l'effectif final du groupe $m\left(\mathrm{~K}_{\mathrm{x}+1}^{\mathrm{m}}\right)$ est lié à l'effectif initial $\left({ }_{\mathrm{j}} \mathrm{K}_{\mathrm{x}}^{\mathrm{m}}\right)$ du même groupe au moyen de :

$$
\mathrm{f}_{\mathrm{x}+1}^{\mathrm{m}}={ }_{\mathrm{i}} \mathrm{K}_{\mathrm{x}}^{\mathrm{m}}-\mathrm{D}_{\mathrm{x}}^{\mathrm{m}}-\mathrm{E}_{\mathrm{x}}^{\mathrm{m}}+\mathrm{I}_{\mathrm{x}}^{\mathrm{m}}-\sum_{\mathrm{n} \neq \mathrm{m}} \mathrm{O}_{\mathrm{x}}^{\mathrm{mn}}+\sum_{\mathrm{n} \neq \mathrm{m}} \mathrm{O}_{\mathrm{x}}^{\mathrm{nm}}
$$

où l'avant-dernier terme représente les mouvements de l'état m vers tous les autres, et le dernier les mouvements en sens inverse.

Comme dans la méthode classique des composantes, les entrées par migration sont incorporées sous forme de nombres absolus alors que les autres éléments le sont sous forme de taux annuels. Tous ces intrants, cependant, sont maintenant affectés d'un superscrit $m$ représentatif du statut conjugal. Dans le cas de la mortalité et de l'émigration, les équations (2) et (3) s'appliquent toujours :

$$
\begin{aligned}
& d_{x}^{m}=D_{x}^{m} / \hat{K}_{x}^{m} \\
& e_{x}^{m}=E_{x}^{m} / k_{x}^{m}
\end{aligned}
$$

où $D_{x}^{m}$ et $E_{x}^{m}$ sont les sorties hors de l'état $m$ dues à la mortalité et à la migration, et $\hat{K}_{x}^{m}$ la population de cet état soumise au risque de décéder et d'émigrer.

Dans le cas des changements de statut conjugal, par contre, on introduit des taux de même nature $\left(o_{x}^{m n}\right)$ en mettant les changements $\left(\mathrm{O}_{x}^{\mathrm{mn}}\right)$ d'un état conjugal $m$ vers un autre $n$ en rapport avec la population soumise au risque de changer d'état, c'est-àdire la population $\hat{\mathrm{K}}_{\mathrm{x}}^{\mathrm{m}}$ de l'état conjugal $\mathrm{m}$, également soumise au risque de mortalité et d'émigration :

$$
\mathrm{O}_{\mathrm{x}}^{\mathrm{mn}}=\mathrm{O}_{\mathrm{x}}^{\mathrm{mn}} / \hat{K}_{\mathrm{x}}^{\mathrm{m}}
$$

Évidemment, par rapport à la méthode classique des composantes, la prise en considération des mouvements entre états conjugaux a le malheur de compliquer les choses. Ainsi, pour chaque groupe ayant un état conjugal donné, supposons que la 
population soumise au risque soit la moyenne arithmétique des effectifs initial $\left({ }_{i} K_{x}^{m}\right)$ et final $\left(\mathrm{K}_{\mathrm{x}+1}^{\mathrm{m}}\right)$. Alors, les équations de taux - les équations (12), (13) et (14) - peuvent, une fois encore, être combinées avec l'équation de mouvements, c'est-à-dire l'équation (11). Il s'ensuit une équation où l'effectif final du groupe $m\left(\mathrm{f}_{\mathrm{f}}^{\mathrm{m}} \mathrm{x}_{\mathrm{x}}\right)$ apparait comme une fonction linéaire non seulement de l'effectif initial du groupe $\left({ }_{i} K_{x}^{m}\right)$ mais aussi des effectifs initiaux $\left({ }_{i} K_{x}^{n}\right)$ et surtout finals $\left({ }_{f} K_{x+1}^{n}\right)$ des autres groupes, soit encore une équation dans laquelle la partie de gauche est une combinaison linéaire des $M$ variables ${ }_{\mathrm{f}} \mathrm{K}_{\mathrm{x}+1}^{\mathrm{m}}$. En d'autres termes, la prise en considération de l'état conjugal conduit à un système linéaire de $M$ équations à $M$ inconnues où les $M$ inconnues sont les $M$ effectifs finals $\left(\mathrm{K}_{\mathrm{x}+1}^{\mathrm{m}}\right)$.

Par référence à la solution de ce système de $M$ équations, nous pourrions évidemment exprimer chacun des effectifs finals $\left(\mathrm{f}_{\mathrm{x}+1} \mathrm{~K}^{\mathrm{m}}\right)$ en fonction des $\mathrm{M}$ effectifs initiaux $\left({ }_{\mathrm{i}} \mathrm{K}_{\mathrm{x}}^{\mathrm{m}}\right)$. Outre qu'elle deviendrait rapidement complexe à mesure que $M$ augmente, la rẻalisation d'une telle tâche ne présente guère d'intérêt, car l'expression complexe ainsi obtenue ne se prêterait à aucune interprétation dêmographique immédiate, même dans le cas où $M$ serait un nombre aussi petit que 2 ou 3 .

Cependant, il est possible d'y voir plus clair grâce à un artifice notationnel consistant à considérer les $\mathrm{M}$ équations obtenues non pas indépendamment mais plutôt de manière jointe et simultanée. Comme nous le montrons ci-dessous, cet artifice va nous conduire à travailler en termes d'entités appelées matrices, pour lesquelles il existe un type de calcul, le calcul matriciel, qui prolonge, de manière plus ou moins simple selon les circonstances, le calcul usuel en termes de scalaires.

\section{Une reformulation matricielle du modèle}

Indiquons ici à l'intention du lecteur profane qu'une matrice (notee dans cet article au moyen de caractères gras de façon à bien marquer la distinction avec un scalaire) n'est autre qu'un tableau rectangulaire regroupant les valeurs prises par une variable dépendant de deux paramètres et tel que l'élément à l'intersection de la émième ligne et de la énième colonne contient la valeur prise par cette variable lorsque le premier paramètre prend sa émième valeur et le second sa énième valeur. Une telle matrice est dite de dimension $M_{1} \times M_{2}$, où $M_{1}$ et $M_{2}$ sont les nombres totaux de valeurs prises par ces deux paramètres. Ajoutons que dans le cas où le deuxième paramètre 
ne prend qu'une seule valeur, c'est-à-dire dans le cas où la variable ne dépend en fait que d'un seul paramètre, la matrice ne comporte qu'une seule colonne (elle est de dimension $M_{1} \times 1$ ) et qu'on lui donne alors le nom de vecteur. En ce cas, nous la notons au moyen de caractères italiques.

Ainsi, considérons la cohorte âgée initialement de $x$ années. Elle consiste en $M$ groupes, un pour chaque état conjugal, dont les effectifs initiaux $\left(_{i} K_{x}^{m}\right)$ respectifs peuvent être rassemblés en un vecteur ${ }_{i} K_{x}$ tel que l'élément situé sur la émième ligne se trouve être ${ }_{i} K_{x}^{m}$ :

$$
{ }_{i} K_{x}=\left[\begin{array}{c}
{ }_{i} K_{x}^{1} \\
{ }_{i} K_{x}^{m} \\
{ }_{i} K_{x}^{M}
\end{array}\right] \begin{gathered}
1 \\
m \\
M
\end{gathered}
$$

De même, les effectifs $\left({ }_{i} I_{x}^{m}\right)$ d'entrées par migration dans chaque état conjugal peuvent être rassemblés en un vecteur ${ }_{i} I_{x}$ tel que l'élément situé sur la émième ligne se trouve être ${ }_{i} I_{x}^{m}$ et les effectifs finals $\left(\mathrm{K}_{\mathrm{x}+1}^{\mathrm{m}}\right)$ dans chaque état conjugal peuvent être rassemblés en un vecteur ${ }_{\mathrm{f}} K_{\mathrm{x}+1}$ formé de manière similaire.

Au cours de la période considérée, les membres de la cohorte se trouvent être redistribués sous l'influence des divers événements démographiques considêrés; cela conduit à la prise en considération de quantités telles la proportion $\mathbf{s}_{\mathrm{x}}^{\mathrm{mn}}$ de ceux qui, parmi les membres de l'effectif initial ${ }_{i} K_{x}^{m}$, survivent dans l'êtat conjugal $n$ à la fin de la période. En ce cas, les diverses valeurs de $s_{x}$, au nombre de $\mathrm{M}^{2}$, peuvent être rassemblêes dans une matrice $\mathbf{s}_{\mathrm{x}}$ où l'élément à l'intersection de la émième ligne et de la énième colonne est $\mathrm{s}_{\mathrm{x}}^{\mathrm{nm}}$ et non $\mathrm{s}_{\mathrm{x}}^{\mathrm{mn}}$, car, de façon générale, les matrices considérées dans cet article sont telles que chaque élément se rapporte à un passage de l'état associé à la colonne dans laquelle il figure à l'état associé à la ligne dans laquelle il figure également :

$$
\mathbf{s}_{\mathrm{x}}=\left[\begin{array}{ccccc}
1 & \ldots & \mathrm{n} & \ldots & \mathrm{M} \\
& \mathrm{s}_{\mathrm{x}}^{11} & & & \mathrm{~s}_{\mathrm{x}}^{\mathrm{M} 1} \\
& & & & \\
& & \mathrm{~s}_{\mathrm{x}}^{\mathrm{nm}} & & \\
& & & \\
& \\
& & & \\
\mathrm{s}_{\mathrm{x}}^{1 \mathrm{M}} & & & \mathrm{s}_{\mathrm{x}}^{\mathrm{MM}}
\end{array}\right] \begin{gathered}
1 \\
\ldots \\
\mathrm{m} \\
\ldots
\end{gathered}
$$


De même, les entrées par migration dans chaque état conjugal se trouvent être redistribuées entre les différents êtats conjugaux avant la fin de la période, donnant ainsi lieu à des quantités $\mathrm{s}_{\mathrm{x}}$ reflétant la proportion des personnes entrées par migration dans l'état $m$ qui survivent à la fin de la période dans l'état $n$. Ces quantités peuvent également être regroupées en une matrice $s_{\mathrm{x}}^{\prime}$.

Brièvement, la notation matricielle permet de réécrire les $M$ équations scalaires issues de la substitution des équations de taux dans les $M$ équations de mouvements sous la forme d'une équation matricielle qui finalement mène à :

$$
{ }_{\mathrm{f}} K_{\mathrm{x}+1}=\mathbf{s}_{\mathrm{xi}} K_{\mathrm{x}}+\mathbf{s}_{\mathrm{x}}^{\prime} I_{\mathrm{x}}
$$

Cette équation, qui ressemble à s'y méprendre à l'équation (4) de la méthode classique des composantes - les scalaires de l'équation (4) ayant fait place à des vecteurs et matrices notés à l'aide des mêmes symboles mais en caractères italiques et gras respectivement -, s'interprète en fait tout comme cette dernière, compte tenu cependant des changements possibles de statut conjugal. Le vecteur ${ }_{\mathrm{f}} K_{\mathrm{x}+1}$ représentatif des effectifs finals se présente donc comme la somme des deux vecteurs que l'on a obtenus, le premier en prémultipliant le vecteur ${ }_{\mathrm{i}} K_{\mathrm{x}}$ des effectifs initiaux par la matrice $\mathbf{s}_{\mathrm{x}}$ des proportions de survie correspondantes, et le second en prémultipliant le vecteur $I_{x}$ des immigrants par la matrice $\mathbf{s}_{x}^{\prime}$ des proportions de survie spécifiques à ceux-ci. Cependant, compte tenu des règles relatives au calcul matriciel, précisons ici que l'équation (15) représente de façon compacte $M$ équations scalaires dont la émième

$$
{ }_{\mathrm{f}} \mathrm{K}_{\mathrm{x}+1}^{\mathrm{m}}=\sum_{\mathrm{n}} \mathrm{s}_{\mathrm{x}}^{\mathrm{nm}}{ }_{\mathrm{i}} K_{\mathrm{x}}^{\mathrm{n}}+\sum_{\mathrm{n}} \mathrm{s}_{\mathrm{x}}^{\prime}{ }^{\mathrm{nm}} I_{\mathrm{x}}^{\mathrm{n}}
$$

indique que l'on obtient l'effectif final du groupe $m$ en sommant les survivants dans l'état $m$ de 1) ceux qui se trouvent initialement dans chacun des états (y compris m) et 2) ceux qui sont entrés par immigration dans chacun des états (y compris m) durant la période considérée.

À noter que les matrices $\mathbf{s}_{\mathrm{x}}$ et $\mathbf{s}_{\mathrm{x}}^{\prime}$ sont données par :

$$
\mathbf{s}_{\mathrm{x}}=\left(\mathbf{I}-0,5 \mathbf{m}_{\mathrm{x}}\right)\left(\mathbf{I}+0,5 \mathbf{m}_{\mathrm{x}}\right)^{-1}
$$

et

$$
\mathbf{s}_{\mathrm{x}}^{\prime}=\left(\mathbf{I}+0,5 \mathbf{m}_{\mathrm{x}}\right)^{-1}
$$

dont la similitude avec les équations scalaires (5) et (7) se rapportant à la méthode classique des composantes est mani- 
feste. En effet, de par une convention notationnelle associée à la présence du terme -1 au dessus de la ligne principale dans (18), la matrice $\mathbf{s}_{x}^{\prime}$ est "l'inverse" de la matrice $\left(\mathbf{I}+0,5 \mathbf{m}_{x}\right)$ de la même façon que dans (7) $\mathrm{s}_{\mathrm{x}}^{\prime}$ était l'inverse de $\left(1+0,5 \mathrm{~m}_{\mathrm{x}}\right)$. De plus, selon (17), la matrice $\mathbf{s}_{\mathbf{x}}$ est le "produit" de la matrice (I - 0,5 $\mathbf{m}_{\mathrm{x}}$ ) par "l'inverse" de la matrice $\left(\mathbf{I}+0,5 \mathbf{m}_{\mathrm{x}}\right)^{2}$ de même que dans (5) $\mathrm{s}_{\mathrm{x}}$ était le produit de $\left(1-0,5 \mathrm{~m}_{\mathrm{x}}\right)$ par l'inverse de $\left(1+0,5 \mathrm{~m}_{\mathrm{x}}\right.$ ) (puisque diviser un nombre par un autre est équivalent à multiplier le premier par l'inverse du second).

Cependant, la comprêhension de (17) et (18) ne saurait être complète sans une explication des matrices $\mathbf{I}$ et $\mathbf{m}_{\mathbf{x}}$. Tout d'abord, précisons que I est une matrice identité de dimension $M \times M$, c'est-à-dire une matrice consistant en $M$ lignes et $M$ colonnes, dans laquelle les éléments diagonaux, ceux qui sont situés sur la diagonale principale joignant le coin supérieur gauche au coin inférieur droit, sont tous égaux à 1 et les éléments non diagonaux, ceux qui sont situés hors de la diagonale principale, sont tous nuls. Elle est appelée matrice identité car, de même que la multiplication de tout scalaire par le scalaire unitaire 1 laisse ce scalaire inchangé, la multiplication de toute matrice par la matrice en question laisse cette matrice inchangée.

Quant à la matrice $\mathbf{m}_{\mathrm{x}}$, elle représente l'extension à la prise en compte du statut conjugal du taux global de sortie. Brièvement, il s'agit d'une matrice de dimension $\mathrm{M} \times \mathrm{M}$ dont les éléments diagonaux ne sont autres que les taux globaux de sortie hors des différents états conjugaux :

$$
m_{x}^{m m}=d_{x}^{m}+e_{x}^{m}+\sum_{n \neq m} o_{x}^{m n}
$$

et dont les éléments non diagonaux sont égaux aux taux de passage d'un état conjugal à un autre, toutefois affectés du signe moins :

$$
m_{x}^{m n}=-o_{x}^{n m}
$$

Observons ici qu'en regroupant les taux de mortalité en une matrice diagonale $\mathbf{d}_{x}$, c'est-à-dire une matrice dont tous les éléments sont nuls hormis ceux de la diagonale contenant les taux de mortalité propres aux divers états conjugaux, et les taux

2 L'expression de $\mathbf{s}_{\mathrm{x}}$ est identique à celle de la matrice de probabilités de transition dérivée par Rogers et Ledent (1976), à ceci près qu'en raison d'un plan d'observation différent une mème matrice (au lieu de deux différentes) apparait dans les deux termes multiplicatifs y figurant. 
d'émigration en une matrice diagonale similaire $\mathbf{e}_{\mathrm{x}}$, on peut alors écrire la matrice $\mathbf{m}_{\mathrm{x}}$ comme suit :

$$
\mathbf{m}_{\mathrm{x}}=\mathbf{d}_{\mathrm{x}}+\mathbf{e}_{\mathrm{x}}+\mathbf{o}_{\mathrm{x}}
$$

où la matrice $o_{x}$ est une matrice contenant les taux de passage d'un état conjugal à un autre :

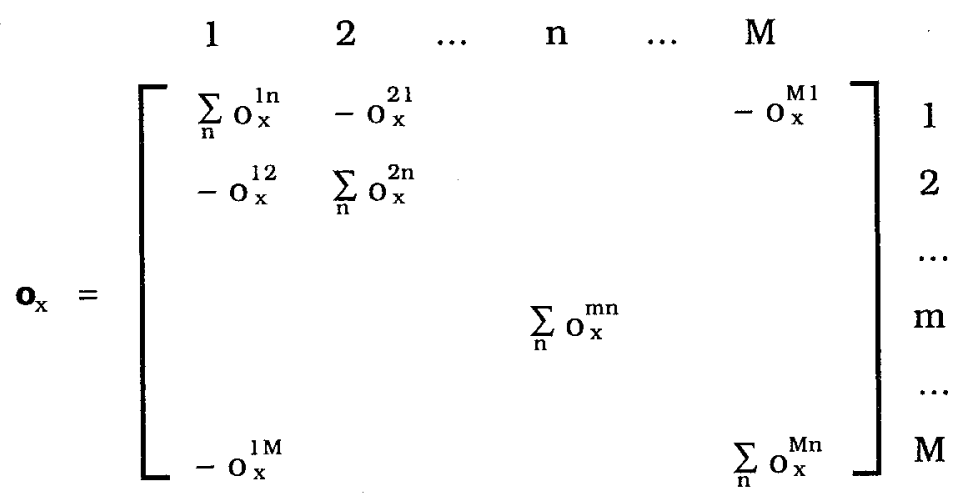

Ainsi l'égalité matricielle (21) génèralise-t-elle l'égalité (6) de la méthode classique des composantes, où $\mathrm{m}_{\mathrm{x}}$ apparaissait comme la somme des taux de mortalité et d'émigration. Cette généralisation se traduit non seulement par le remplacement de la somme de deux scalaires, $d_{x}$ et $e_{x}$, par la somme de deux matrices, $\mathbf{d}_{x}$ et $\mathbf{e}_{x}$, mais aussi par l'ajout au résultat d'une matrice $\mathbf{o}_{\mathrm{x}}$ contenant les taux se rapportant aux changements de statut conjugal. Cette dernière matrice comprend $M$ colonnes telles que dans chacune les éléments situés hors de la diagonale principale sont les taux de passage de l'état conjugal associé vers tous les autres, toutefois précédés du signe moins, alors que l'élément situé sur la diagonale principale n'est autre que la somme de ces taux ${ }^{3}$.

Avant d'en terminer, il reste à examiner le cas du premier groupe d'âge $(x=-1)$ par le biais duquel entre la natalité. À nouveau, les développements afférents au cas de la méthode classique des composantes se généralisent facilement à l'aide

3 Le lecteur s'étonnera sans doute du fait que dans la matrice $\boldsymbol{o}_{\mathrm{x}}$ les éléments correspondant à des sorties soient affectés d'un signe positif et les éléments correspondant à des entrées soient affectés d'un signe négatif. Il en est ainsi parce que la matrice $\mathbf{m}_{\mathbf{x}}$ est construite comme une extension directe du taux global de sortie $\mathrm{m}_{\mathrm{x}}$ de la méthode classique des composantes. Alternativement, nous aurions pu généraliser $m_{x}^{\prime}=-m_{x}$ en une matrice $\mathbf{m}_{x}^{\prime}=-\mathbf{m}_{x}$ et ainsi attribuer aux entrées (hors diagonale) un signe positif et aux sorties (dans la diagonale) un signe négatif. 
du calcul matriciel. De fait, si l'on introduit les naissances au moyen de taux de fécondité par état conjugal

$$
\mathbf{f}_{\mathrm{x}}^{\mathrm{m}}=\mathrm{N}_{\mathrm{x}}^{\mathrm{m}} / \mathrm{R}_{\mathrm{x}}^{\mathrm{m}}
$$

l'équation (9) de la méthode classique des composantes cède la place à

$$
{ }_{\mathrm{f}} K_{0}=\mathbf{s}_{-1}^{\prime}\left(\mathbf{J} N_{.}+L_{-1}\right)
$$

où $\mathbf{s}_{-1}^{\prime}$ est la matrice de proportions de survie liée à $\mathbf{m}_{-1}$ par la formule générale liant $\mathbf{s}_{\mathrm{x}}^{\prime} \mathbf{a} \mathbf{m}_{\mathrm{x}}$; cette matrice de proportions se trouve donc être appliquée d'une part au vecteur des nouveaunés

$$
N_{.}=\sum_{\mathrm{x}} \mathbf{f}_{\mathrm{x}}\left(\left(1+\mathbf{s}_{\mathrm{x}}\right)_{\mathrm{i}} K_{\mathrm{x}}+I_{\mathrm{x}}\right) / 2
$$

où $\mathbf{f}_{x}$ est une matrice diagonale regroupant les taux de fécondité, et d'autre part au vecteur des immigrants $I_{-1}$ relatif au premier groupe d'âge.

L'équation matricielle (23) se présente bien comme une généralisation de l'équation scalaire (9), mais à l'inclusion près d'une matrice $\mathbf{J}$ dont le rôle est de rediriger les naissances selon l'état conjugal de la mère vers l'état conjugal unique caractérisant les nouveau-nés. Une telle matrice est constituée d'éléments nuls à l'exception de ceux qui sont situés sur la ligne correspondant à l'état conjugal des nouveau-nés, tous égaux à 1 .

Pour résumer, l'introduction du statut conjugal dans la méthode des composantes conduit à suivre non seulement l'effectif de chaque cohorte mais aussi la distribution de cet effectif entre les états conjugaux. Elle conduit à un ensemble d'équations interdépendantes - dans la mesure où une sortie d'un état conjugal est aussi une entrée dans un autre état - mais difficilement maniables sur le plan analytique. Fort heureusement, le recours à une notation matricielle permet de pallier cette difficulté. Comme nous l'avons vu plus haut, les formules afférant à la méthode classique des composantes demeurent valides en des termes fort semblables dans la mesure où des matrices (et vecteurs) viennent se substituer aux scalaires $y$ figurant, tout en se parant d'une interprétation démographique qui généralise de manière immédiate celle de ces derniers. Alors que, dans la méthode classique des composantes, l'effectif final se déduit de l'effectif initial sur la base de formules simples impliquant les taux globaux de sortie $\left(m_{x}=d_{x}+e_{x}\right)$ d'une part et les immigrants $\left(I_{x}\right)$ d'autre part, dans le cas présent le vecteur des effectifs finals se déduit de celui des effectifs initiaux sur la base de formules similaires - hormis la présence de $\mathbf{J}$ dans l'équation (23) - impliquant une matrice de taux de 
sortie ( $\left.\mathbf{m}_{\mathrm{x}}=\mathbf{d}_{\mathrm{x}}+\mathbf{e}_{\mathrm{x}}+\mathbf{o}_{\mathrm{x}}\right)$ d'une part et un vecteur d'immigrants $\left(I_{\mathrm{x}}\right)$ d'autre part. Particulièrement importante est la matrice $\mathbf{o}_{\mathrm{x}}$ qui entre dans la définition de $\mathbf{m}_{\mathrm{x}}$. Incorporant les taux de passage d'un état à un autre (précédés du signe moins) hors diagonale et les taux totaux de changement de statut conjugal dans la diagonale, c'est elle qui de fait sous-tend la redistribution des membres de toute cohorte entre les différents états conjugaux.

\section{LA DÉTERMINATION DU NOMBRE ET DE L'ÂGE DES ENFANTS PRÉSENTS}

Ayant traité jusqu'ici de la projection des effectifs de femmes selon le statut conjugal, nous passons à l'étape suivante, qui consiste à désagréger ces effectifs selon le nombre d'enfants présents auprès d'elles. La démarche à suivre à cet effet s'impose d'elle-même. Dans un premier temps, nous chercherons à déterminer pour chaque femme le nombre d'enfants déjà nés, c'est-àdire la parité. Puis, dans un deuxième temps, nous passerons de ce nombre à celui des enfants effectivement présents en traitant de manière explicite de leur absence possible. Enfin, dans un troisième temps, nous élargirons la procédure à la détermination de l'âge des enfants selon le rang.

\section{Extension du modèle à la prise en compte de la parité}

L'élargissement de la méthode classique des composantes à la prise en compte du statut conjugal n'est pas spécifique à ce statut démographique. Si, au lieu de considèrer le statut conjugal, nous considérions une autre variable de statut démographique comme la parité, le statut d'activité, etc., la formulation du modèle ne différerait pas de celle que nous avons présentée ${ }^{4}$ car l'élément qui importe ici est bien le fait de désagréger les cohortes en plusieurs groupes selon la variable de statut considérée, et non pas la nature même de cette variable.

Par ailleurs, si la méthode classique des composantes est élargie à la prise en compte de deux ou plusieurs variables de statut démographique, la formulation matricielle décrite plus

4 Signalons toutefois que, dans le cas du premier groupe d'áge $(x=-1)$, une modification de la matrice $\boldsymbol{J}$ est nécessaire si l'état attribué aux nouveaunés n'a pas une valeur unique. Ainsi, si la variable de statut démographique est la région de résidence, les nouveau-nés se voient normalement attribuer comme région de résidence celle de leur mère, de sorte que la matrice $\mathbf{J}$, qui n'est autre que la matrice identité de même dimension, peut être omise. 
haut demeure valide. Seule la dimension des vecteurs et matrices impliqués change au rythme des croisements de statuts démographiques effectués. Ainsi, l'ajout de la parité avec $\mathrm{P}$ états $-\mathrm{p}(=0,1,2, \ldots, \mathrm{P}-2, \mathrm{P}-1$ et plus $)$ étant la parité ou nombre d'enfants déjà nés pour une femme classée en l'état $\mathrm{p}+1$ - au statut conjugal avec $M$ états conduit à utiliser des vecteurs de dimension $\mathrm{MP}$ et des matrices de dimension $\mathrm{MP} \times \mathrm{MP}$.

Par suite, le vecteur d'immigrants $I_{x}$ apparaît constitué de $P$ segments ou sous-vecteurs $\bar{I}_{\mathrm{x}}^{\mathrm{p}}$ similaires au vecteur $I_{\mathrm{x}}$ de la section précédente dans la mesure où ces sous-vecteurs contiennent les effectifs d'immigrants par état conjugal mais pour une parité donnée. De même, les matrices $\mathbf{d}_{x}$ et $\mathbf{e}_{\mathrm{x}}$ apparaissent constituées de sous-matrices diagonales $\overline{\mathbf{d}}_{\mathrm{x}}^{\mathrm{p}}$ et $\mathbf{e}_{\mathrm{x}}^{-\mathrm{p}^{\mathrm{x}}}$ similaires aux matrices diagonales $\mathbf{d}_{\mathrm{x}}$ et $\mathbf{e}_{\mathrm{x}}$ de la section précédente, c'est-à-dire qu'elles contiennent les taux de mortalité et d'émigration par état conjugal, mais pour une parité donnée.

Signalons que cette description de $I_{x}, \mathbf{d}_{x}$ et $\mathbf{e}_{\mathrm{x}}$ fait passer la parité avant le statut conjugal. Nous aurions pu aussi bien adopter la démarche inverse et spécifier $I_{x}, \mathbf{d}_{x}$ et $\mathbf{e}_{\mathrm{x}}$ en faisant passser le statut conjugal avant la parité. Cependant, comme la parité reflète un statut démographique évoluant de manière séquentielle sans possibilité de retour dans un état antërieur, il y a intérêt à faire passer la parité avant l'état conjugal car, contrairement au traitement alternatif, ce traitement mène à une spécification simple et parlante de la matrice $\mathbf{o}_{\mathrm{x}}$.

Pour voir cela, découpons la matrice $\boldsymbol{o}_{\mathrm{x}}$ en sous-matrices $o_{x}(p, q)$ formées des éléments figurant à l'intersection des $M$ lignes correspondant à la p-ième catégorie de parité et des $\mathrm{N}$ colonnes correspondant à la q-ième catégorie de parité. $\mathrm{Vu}$ que tout changement hors de la parité p-1 s'effectue uniquement vers la parité $p$, il s'ensuit que les sous-matrices $\mathbf{o}_{x}(p, q)$ sont nulles (ne contiennent que des éléments nuls) à moins que $\mathrm{p}$ soit égal à $\mathrm{q}$ ou $\mathrm{q}+1$.

Par construction, la sous-matrice $\mathbf{o}_{x}(p, p)$ est une matrice rreflétant deux événements possibles pour une femme de parité $\mathrm{p}-1$ et d'état conjugal $\mathrm{m}$ : un changement d'état conjugal et la naissance du p-ième enfant. Elle s'exprime donc comme la somme de deux sous-matrices où la première, $\overline{\mathbf{o}}_{\mathrm{x}}^{\mathrm{p}-1}$, est la matrice $o_{x}$ de la section précédente mais pour une parité donnée, et où la seconde, $\overline{\mathbf{f}}_{\mathrm{x}}^{\mathrm{p}-1}$, est la matrice $\mathbf{f}_{\mathrm{x}}$ de la section précédente mais également pour une parité donnée :

$$
\mathbf{o}_{x}(\mathrm{p}, \mathrm{p})=\overline{\mathbf{o}}_{\mathrm{x}}^{\mathrm{p}-1}+\overline{\mathbf{f}}_{\mathrm{x}}^{\mathrm{p}-1}
$$


Dans le cas de la parité la plus élevée, (25) se réduit au premier terme $\widetilde{\boldsymbol{o}}_{\mathrm{x}}^{\mathrm{P}-1}$ vu que le second terme est nul (la catégorie de parité demeurant inchangée).

Et, puisque la naissance du p-ième enfant est non seulement une sortie de la parité $\mathrm{p}-1$ mais aussi une entrée dans la parité $\mathrm{p}$, nous avons bien entendu pour tout $\mathrm{p}<\mathrm{P}$

$$
\mathbf{o}_{\mathrm{x}}(\mathrm{p}+1, \mathrm{p})=-\overline{\mathbf{f}}_{\mathrm{x}}^{\mathrm{p}-1}
$$

\section{Le principe de passage de la parité au nombre d'enfants présents}

Afin de passer du nombre d'enfants mis au monde au nombre d'enfants effectivement présents, il nous faut déterminer dans quelle mesure les enfants demeurent auprès de leur mère. Comme nous l'avons évoqué, la méthodologie sous-jacente au modèle utilisé ne permet pas de suivre les événements démographiques de deux ou plusieurs personnes à la fois. Il est néanmoins possible de s'affranchir de cette limite en ayant recours à un subterfuge initialement décrit par Bongaarts (1987) et dont nous nous efforçons ci-après de démonter les rouages.

Supposons provisoirement que seul un décès puisse être la cause de l'absence d'un enfant auprès de sa mère, du moins tant que l'âge de l'enfant demeure en dessous d'un seuil égal à $\mathrm{X}$ années. Et de plus, supposons qu'au delà de ce seuil tout enfant est réputé avoir quitté sa mère. Dans ces conditions, la probabilité qu'un enfant né en une année $(t, t+1)$ soit présent à l'instant $T$ aux côtés de sa mère (toujours en vie) est égale à la probabilité $r_{x-0,5}$ (notée tout simplement $r$ par la suite) de ne pas décéder avant l'âge $x-0,5$ (où $x=T-t$ ), tant que $x$ est inférieur ou égal à $X$, alors que, pour tout $\mathrm{X}$ supérieur à $\mathrm{X}$, elle est nulle. En d'autres termes, pour toute cohorte de nouveau-nés, nous connaissons le nombre de ceux qui sont effectivement présents auprès de leur mère sur les $X$ années suivant la naissance. Il s'ensuit que, pour toute cohorte de femmes nées en une même année, la détermination endogène des naissances dans le modèle de projection conduit immédiatement à la connaissance en tout temps des enfants qui survivent aux côtés de leur mère.

Cependant, pour connaître tous les enfants survivant auprès de leur mère à un instant donné, il faut une détermination endogène des naissances sur les $X$ années précédentes. C'est pourquoi la mise en œuvre du modèle de projection doit débuter non pas, comme c'est normalement le cas, au terme de la période d'observation, mais bien $\mathrm{X}$ années plus tôt. Pour fixer les 
idées, supposons que partant de l'année 1991 on veuille projeter le nombre d'enfants effectivement présents auprès de leur mère. Si de plus le seuil X est fixé à 18 ans, alors le modèle est à mettre en œuvre à partir de 1973 (si la connaissance des enfants présents est désirée pour l'année de départ) ou de $1973+$ y (si la première année pour laquelle la connaissance des enfants présents est désirée se situe y années au delà de 1991).

Jusqu'ici, notre raisonnement sur la survie des enfants auprès de leur mère a été fait indépendamment de celle-ci et donc, pour chaque enfant, indépendamment de la survie de ses frère(s) et sœur(s) éventuels, de sorte qu'il ne mène pas encore à la détermination du nombre d'enfants effectivement présents. Pour y arriver, étant donné que les enfants sont issus de cohortes différentes dont on ne peut considérer simultanément l'évolution, la seule façon concevable d'opérer est :

- d'appliquer les probabilités de survie aux enfants dès leur naissance et donc

- de projeter indépendamment le nombre d'enfants effectivement présents pour chacune des années-horizons, ou années pour lesquelles ce nombre est désiré.

Pour en revenir à l'exemple qui vient d'être considéré, si l'objectif est de déterminer les enfants effectivement présents à cinq ans d'intervalle entre 1991 et 2016, nous devons mettre le modèle en œuvre à six reprises : une première fois sur la période 1973-1991, une seconde fois sur la période 1978-1996 et ainsi de suite jusqu'à une sixième fois sur la période $1998-2016^{5}$.

Ayant indiqué le principe général autorisant la détermination du nombre d'enfants effectivement présents, il nous reste à montrer comment la mettre en œuvre. Faute de place, nous ne pouvons le faire de manière détaillée et donc ci-dessous nous nous contenterons d'apporter deux précisions susceptibles d'aider le lecteur à concevoir cette mise en œuvre.

Premièrement, en nous référant à l'archétype qui nous a permis plus haut d'élargir la méthode des composantes à la prise en compte de l'état conjugal et de la parité, nous pouvons

5 Évidemment, une telle multiplication de projections pose un problème intéressant de compatibilité ayant trait, d'une part, aux résultats des six projections entre eux et, d'autre part, aux valeurs projetées pour 1991 et à celles tirées du recensement correspondant. Loin d'être trivial, ce problème est cependant soluble. Brièvement, la solution consiste à redistribuer sur l'ensemble de la période 1971-1991 - selon des modalités qui seraient trop longues à expliquer - les écarts constatés entre les valeurs projetées et observées de 1991 et, pour chaque année, à les inclure dans un vecteur de rêsidus s'ajoutant à celui des immigrations. 
affirmer que la détermination du nombre d'enfants effectivement présents peut se réaliser sur la base du modèle précédent après introduction d'un croisement par une troisième variable reflétant le nombre d'enfants présents. Vu que le nombre d'enfants effectivement présents, noté c ci-après, est inférieur ou égal à la parité $p$ de la mère, le nombre d'états supplémentaires entraînés par l'inclusion de cette troisième variable de statut démographique n'est pas directement proportionnel à $\mathrm{P}$. De fait, quant la parité augmente d'une unité, le nombre d'états ajoutés est précisément égal à la nouvelle valeur de cette variable de sorte que le nombre total d'états ajoutés est égal à $1+2+\ldots+P$. Comme la somme des $n$ premiers entiers est égale à $n(n+1) / 2$, il s'ensuit que les vecteurs considérés ici sont de dimension $\mathrm{N}$ et les matrices de dimension $\mathrm{N} \times \mathrm{N}$, où $\mathrm{N}=\mathrm{MP}(\mathrm{P}+1) / 2$.

Deuxièmement, l'application des probabilités de survie aux enfants dès leur naissance n'a aucun impact sur la prise en compte des sorties hors de chaque état puisque, que le nouveauné survive ou non jusqu'à l'année-horizon considérée, il y a de toutes manières sortie de l'état considéré (en raison du changement de parité sous-jacent). Ainsi, si l'on ordonne les trois variables de statut démographique de manière à intercaler le nombre d'enfants présents entre la parité et l'état conjugal, la sous-matrice $\mathbf{o}_{\mathrm{x}}(\mathrm{p}, \mathrm{p})$ de dimension $\mathrm{M} \times \mathrm{M}$ définie précédemment fait place à une sous-matrice élargie de dimension $\mathrm{Mp} \times \mathrm{Mp}$ et constituée de blocs diagonaux tous identiques à elle-même. Cependant, ce résultat ne s'applique pas dans le cas de la parité plus élevée car, si la sous-matrice $\mathbf{o}_{\mathrm{x}}(\mathrm{P}, \mathrm{P})$ ne compte qu'un terme se rapportant aux changements de statut conjugal, son extension dans le présent contexte nécessite que l'on ajoute à la sous-matrice généralisant les changements de statut conjugal une autre sous-matrice liée à l'augmentation possible d'une unité du nombre d'enfants présents, bien que la catégorie de parité demeure inchangée.

Par contre, l'application des probabilités de survie affecte les entrées, ce qui conduit à remplacer, du moins à ce niveau, chacun des taux de fécondité $\mathrm{f}_{\mathrm{x}}^{(\mathrm{m})}$ par deux taux associés se rapportant l'un aux nouveau-nés qui survivent jusqu'à l'annéehorizon considérée et l'autre à ceux qui ne survivent pas jusqu'à cet instant. Leurs valeurs se déduisent aisément de $\mathrm{f}_{\mathrm{x}}^{\mathrm{p}(\mathrm{m})}$ au moyen d'une multiplication par $r$ et (1-r) respectivement. Il s'ensuit que la sous-matrice $\mathbf{o}_{\mathrm{x}}(\mathrm{p}+1, \mathrm{p})$ de dimension $\mathrm{M} \times \mathrm{M}$ considérée précédemment fait place à une sous-matrice élargie 
de dimension $M(p+1) \times M p$, constituée de sous-matrices nulles hors de deux "obliques" : celle qui part du coin supérieur gauche et celle, juste en-dessous, qui aboutit au coin inférieur droit :

$$
\begin{aligned}
& 1 \\
& 2 \\
& \text {.. } \mathrm{p}-1 \\
& \mathrm{p} \\
& {[}
\end{aligned}
$$

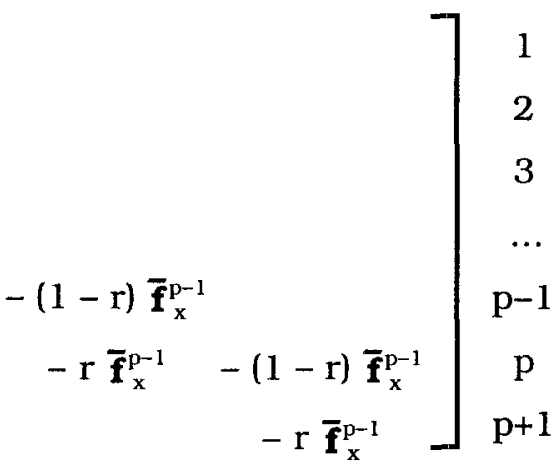

Il s'ensuit par la même occasion que le second terme de la sous-matrice $o_{x}(P, P)$, de dimension $M p \times M p$ se présente ainsi :

1

$\Gamma$
2

$$
r \overline{\mathbf{f}}_{x}^{\mathrm{P}}
$$

$-r \overline{\mathbf{f}}_{x}^{P}$

$$
\begin{gathered}
r \overline{\mathbf{f}}_{x}^{\mathrm{P}-1} \\
-\mathbf{r} \overline{\mathbf{f}}_{x}^{\mathrm{P}-1}
\end{gathered}
$$

$\mathrm{P}-1$

$\mathrm{P}$

où le terme en bas et à droite de la "diagonale" est bien nul puisque, en ce cas, la catégorie de parité et celle relative au nombre d'enfants sont bloquées à leur valeur maximale.

\section{La détermination du nombre d'enfants présents : présentation résumée et discussion}

À ce stade, il n'est sans doute pas inutile de reprendre de manière plus globale le modèle auquel nous avons abouti et par ce biais d'apporter quelques précisions supplémentaires à propos de sa formulation. D'un point de vue strictement formel, le modèle complet se présente comme une généralisation immédiate de la méthode classique des composantes. Elle s'appuie sur deux équations - les équations (15) pour le groupe d'âge 
typique et (23) pour le premier groupe d'âge - similaires aux équations (4) et (9) de la méthode classique. Les scalaires impliqués dans (4) et (9) font place dans (15) et (23) à des vecteurs et matrices les généralisant dans le contexte de changements possibles de statut au regard de trois caractéristiques : le statut conjugal, la parité et le nombre d'enfants présents.

Les équations (15) et (23) mettent en jeu un vecteur $I_{\mathrm{x}}$ d'immigrants, sur lequel nous reviendrons un peu plus tard, et une matrice de taux de sortie $\mathbf{m}_{\mathrm{x}}=\mathbf{d}_{\mathrm{x}}+\mathbf{e}_{\mathrm{x}}+\mathbf{o}_{\mathrm{x}}$ où les deux premiers éléments, $\mathbf{d}_{x}$ et $\mathbf{e}_{x}$, sont des matrices diagonales de taux de mortalité et d'émigration hors des divers états issus du croisement des trois variables de statut démographique et le troisième, $\mathbf{o}_{\mathrm{x}}$, est une matrice contenant les taux de passage entre ces états. Si les trois variables de statut sont ainsi ordonnées : 1) la parité $\mathrm{p}, 2$ ) le nombre $\mathrm{c}$ d'enfants présents et 3) le statut conjugal $\mathrm{m}$, alors la matrice $\mathbf{o}_{\mathrm{x}}$ se présente comme suit (dans le cas particulier où $\mathrm{p}=5$ ) :

\begin{tabular}{|c|c|c|c|c|c|c|c|c|c|c|c|c|c|c|c|}
\hline & & $\begin{array}{l}0 \\
0\end{array}$ & $\begin{array}{ll}1 & \\
0 & 1\end{array}$ & $\begin{array}{l}2 \\
0\end{array}$ & 1 & 2 & $\begin{array}{l}3 \\
0\end{array}$ & 1 & 2 & 3 & $\begin{array}{l}4 \\
0\end{array}$ & 1 & 2 & 3 & 4 \\
\hline 0 & 0 & $\mathbf{a}$ & & & & & & & & & & & & & \\
\hline 1 & $\begin{array}{l}0 \\
1\end{array}$ & $\begin{array}{l}\text { b } \\
\text { c }\end{array}$ & $\mathbf{a}$ & & & & & & & & & & & & \\
\hline 2 & $\begin{array}{l}0 \\
1 \\
2\end{array}$ & & $\begin{array}{ll}\text { b } & \\
\text { c } & \text { b } \\
& \text { c }\end{array}$ & $\mathbf{a}$ & $\mathbf{a}$ & $\mathbf{a}$ & & & & & & & & & \\
\hline 3 & $\begin{array}{l}0 \\
1 \\
2 \\
3\end{array}$ & & & $\begin{array}{l}\mathbf{b} \\
\mathbf{c}\end{array}$ & $\begin{array}{l}\text { b } \\
\text { c }\end{array}$ & b & $\mathbf{a}$ & $\mathbf{a}$ & a & $\mathbf{a}$ & & & & & \\
\hline 4 & $\begin{array}{l}0 \\
1 \\
2 \\
3 \\
4\end{array}$ & & & & & & $\begin{array}{l}\text { b } \\
\text { c }\end{array}$ & $\begin{array}{l}\text { b } \\
\text { c }\end{array}$ & & c & $\begin{array}{l}\mathbf{d} \\
\mathbf{c}\end{array}$ & $\begin{array}{l}\text { d } \\
\text { c }\end{array}$ & $\begin{array}{l}\text { d } \\
\text { c }\end{array}$ & d & e \\
\hline
\end{tabular}


Précisons que :

- cette matrice est de dimension $\mathrm{N} \times \mathrm{N}$, où $\mathrm{N}$ est un multiple de $M$ (le nombre d'états conjugaux) égal à 15 (vu que pour $\mathrm{P}=5, \mathrm{P}(\mathrm{P}+1) / 2$ vaut précisément 15$)$,

- la ligne la plus haute et la colonne la plus à gauche indiquent la parité tandis que la ligne située immédiatement en dessous et la colonne située immédiatement à droite se rapportent au nombre d'enfants présents et

- seules sont représentées les sous-matrices non nulles, qui, dans chaque "colonne" relative au croisement d'une parité et d'un nombre d'enfants présents donnés, s'expriment ainsi :

$$
\begin{aligned}
& \mathbf{a}=\overline{\mathbf{o}}_{\mathrm{x}}^{\mathrm{p}-1}+\overline{\mathbf{f}}_{\mathrm{x}}^{\mathrm{p}-1} \\
& \mathbf{b}=-(1-\mathrm{r}) \overline{\mathbf{f}}_{\mathrm{x}}^{\mathrm{p}-1} \\
& \mathbf{c}=-\mathrm{r} \overline{\mathbf{f}}_{\mathrm{x}}^{\mathrm{p}-1}
\end{aligned}
$$

Cependant, dans le cas de la parité la plus élevée, la sousmatrice a est remplacée par :

$$
\mathbf{d}=\overline{\mathbf{o}}^{\mathrm{P}-1}+\mathbf{r} \overline{\mathbf{f}}_{\mathrm{x}}^{\mathrm{P}-1}
$$

et, dans le cas où le nombre des enfants présents est aussi égal à sa valeur maximale, par

$$
\mathbf{e}=\overline{\mathbf{o}}_{\mathrm{x}}^{\mathrm{P}-1}
$$

D'un point de vue pratique, l'application de ce modèle de manière à déterminer le nombre d'enfants effectivement présents est également plus complexe que celle de la méthode classique des composantes. Contrairement à cette dernière, qui requiert une mise en œuvre unique en partant de la population en fin de période d'observation, elle nécessite :

- d'une part que l'on mette en œuvre le modèle non pas une seule fois mais autant de fois qu'il y a d'années-horizons et

- d'autre part que l'on réalise chaque mise en œuvre en partant non pas de la population de la fin de la période d'observation mais bien de la population observée (ou éventuellement projetée si l'horizon est suffisamment éloigné) $\mathrm{X}$ années avant l'année-horizon.

Des résultats générés par chaque mise en œuvre, seuls ceux qui se rapportent à l'année-horizon nous sont utiles car, il est bon ici de le remarquer, la variable c reflète le nombre 
d'enfants présents non pas en l'année atteinte dans le processus de projection mais bien ce nombre au terme de la projection, c'est-à-dire en l'année-horizon sélectionnée. Une telle remarque mène d'ailleurs à s'interroger sur les valeurs prises par les vecteurs $I_{x}$ d'immigrants. En effet, ces vecteurs, qui étaient exogènes tant que la méthode classique des composantes était élargie à la prise en compte du statut conjugal et de la parité, ne le sont plus à partir du moment où l'on inclut le nombre d'enfants effectivement présents car, comme nous venons de l'entrevoir, ce nombre se rapporte non pas à l'année d'immigration mais au terme de la projection! Si l'âge des enfants qui accompagnent les immigrantes ayant un statut conjugal et une parité donnés est connu, alors l'application de probabilités de survie jusqu'à l'année-horizon autorise une détermination immédiate des vecteurs $I_{x}$ au fil des années. Par contre, s'il n'est pas connu, nous nous trouvons en face d'un problème ardu mais soluble, que faute de place nous ne pouvons traiter ici.

Enfin, pour en terminer avec cette sous-section, nous devons faire deux remarques finales. Premièrement, le nombre d'enfants peut être précisé selon le sexe grâce 1) à l'introduction d'un paramètre de répartition des naissances entre sexes masculin et féminin et 2) à l'utilisation de probabilités de survie spécifiques à chacun des deux sexes. Deuxièmement, il nous reste à lever l'hypothèse provisoire adoptée plus haut suivant laquelle la mortalité est le seul facteur susceptible d'entraîner une absence du foyer maternel (tant que le seuil de $\mathrm{X}$ années n'est pas atteint). Pour cela, supposons que parmi les enfants survivant jusqu'à l'âge $\mathrm{x}-0,5$ l'on puisse observer la proportion $t_{x-0,5}$ de ceux qui demeurent auprès de leur mère ${ }^{6}$. Alors la procédure décrite précédemment pour déterminer le nombre d'enfants présents demeure valide à condition que l'on remplace la probabilité de survie $r_{x-0,5}$ par le produit $r_{x-0,5} t_{x-0,5}$. Alternativement, si les sorties et entrées peuvent être observées sous la forme de taux, ce produit peut être remplacé par la probabilité de survie auprès de la mère dérivée d'une table de mortalité multidimensionnelle à deux états (vie au foyer maternel, vie hors du foyer maternel).

6 Au lieu de présumer qu'un enfant quitte sa mère pour ne plus revenir, le recours à une telle proportion présente l'avantage de refléter le solde des mouvements de sortie et de retour au foyer maternel que peut effectuer un enfant tant que $\mathrm{x}$ demeure inférieur ou égal à $\mathrm{X}$. 


\section{La détermination de l'âge des enfants}

La détermination de l'âge des enfants est un objectif plus ou moins facile à atteindre selon l'ampleur du niveau de détail recherché. Ainsi, pour s'en tenir à un niveau minimal n'impliquant aucune extension nouvelle de notre modèle, il est relativement simple d'établir combien de femmes ayant un état conjugal et une parité donnés ont auprès d'elles des enfants appartenant à une catégorie d'âge donnée, par exemple des enfants en bas âge. Le nombre de femmes en question peut même être désagrégé sans peine selon le nombre d'enfants dans la catégorie considérée.

De façon pratique, afin de déterminer l'âge des enfants pour chaque année-horizon, il faut répéter l'application du modèle, en fait autant de fois qu'il existe de groupes d'âge d'enfants, en prenant soin chaque fois de modifier les proportions de survie de manière appropriée. Supposons, pour fixer les idées, que soient retenus $n$ groupes d'âge $\mathrm{A}, \mathrm{B}, \mathrm{C}, \ldots$ (A étant le groupe le plus jeune) tels que $X_{A}, X_{B}, X_{C}, \ldots$ dénotent les bornes d'âge supérieures respectives. Alors, une première application du modèle précédent avec les probabilités de survie des enfants prises égalées à zéro pour tout âge plus grand que $\mathrm{X}_{\mathrm{A}}$ livre la distribution (en l'année-horizon considérée) des femmes ayant un état conjugal et une parité donnés selon le nombre d'enfants appartenant au groupe d'âge A. De même, une seconde application du modèle avec les probabilités de survie égalées à zéro pour tout âge compris entre $X_{A}$ et $X_{B}$ livre la distribution de ces mêmes femmes selon le nombre d'enfants appartenant au groupe d'âge B. Et ainsi de suite...

\section{PARACHẼVEMENT DU MODELLE}

Quoique digne d'un intérêt propre, la projection des effectifs de la population féminine selon 1) le statut conjugal et 2) le nombre et l'âge des enfants ne nous intéresse qu'en tant que tremplin menant à la projection des effectifs de familles selon leurs principales caractéristiques. Aussi cette section n'a-t-elle d'autre objet que de montrer comment effectuer le passage des effectifs de femmes selon leurs statuts démographiques à ceux des familles selon leurs caractéristiques principales.

Ce passage, on s'en doute, s'appuie sur 1) le statut conjugal et 2) la présence d'enfants. Tout d'abord, le statut conjugal, dont le propre est de refléter la modalité de vie des femmes concernées, 
permet de redistribuer celles-ci entre les divers types de famille possibles. Ignorée jusqu'ici car n'ayant aucune influence sur la formulation du modèle présentée dans les trois sections précédentes, la nomenclature des états représentatifs du statut conjugal présente ici un intérêt de première importance. Naturellement, elle est étroitement liée au contexte d'application mais, quel que soit celui-ci, il est inévitable que la nomenclature utilisée repose sur deux grands types d'états, séparant les femmes qui vivent en union, légale ou non peu importe, de celles qui vivent sans conjoint, légal ou non. C'est pourquoi, des effectifs de femmes projetés par le modèle, découlent directement les effectifs de celles qui vivent en union, correspondant à autant de familles époux-épouse (pour employer le jargon de Statistique Canada), ainsi que les effectifs de celles qui vivent sans conjoint (mais pas nécessairement dans des familles).

Ensuite, la présence d'enfants, quel qu'en soit le nombre, conduit à diviser chacun de ces deux groupes de femmes en deux sous-groupes avec ou sans enfant. Nous sommes ainsi en mesure, dans le premier cas, de séparer les familles biparentales des familles époux-épouse sans enfant et, dans le second, d'identifier les familles monoparentales à parent féminin (les femmes n'ayant ni conjoint, ni enfant vivant clairement hors famille).

Bien entendu, une fois effectué le passage des femmes aux familles comme nous venons de le décrire, nous ne sommes pas encore au bout de nos peines, car la typologie des familles qui en résulte n'est pas très réaliste. D'une part, nous n'y retrouvons pas les familles monoparentales à parent masculin; d'autre part, les familles biparentales s'y présentent comme des familles où tous les enfants sont issus de la mère, alors qu'en réalité nombre de familles biparentales sont des "familles reconstituées" où quelques-uns sinon tous les enfants peuvent être issus d'une précédente union contractée par le conjoint masculin. La nécessité de prendre en compte ces situations réelles appelle donc un amendement du modèle, que nous nous employons à décrire dans le reste de cette section.

En premier lieu, notons que de telles familles peuvent se former en deux occasions :

- lorsque, après le décès d'une femme, la garde de ses enfants passe à un homme pouvant être son conjoint, si elle vivait en union, ou alors, si elle vivait seule, un ex-conjoint ou bien même, si elle n'avait jamais contracté d'union, le père des enfants; et 
- lorsque, après une séparation, les enfants sont retirés à leur mère pour être confiés au conjoint dont elle se sépare ou peut-être au père si celui-ci n'était pas le conjoint.

Dans le premier cas, cependant, une famille monoparentale à parent masculin ne se forme que si l'homme auquel passent les enfants vit seul (sans conjointe). Sinon, les enfants entrent en fait dans une famille époux-épouse dite reconstituée selon la définition que nous avons faite un peu plus haut de ce terme.

Il s'ensuit que la possibilité de déterminer les effectifs de familles monoparentales à parent masculin et, par la même occasion, celle de séparer les familles reconstituées des autres familles époux-épouse obligent à suivre l'évolution des enfants séparés de leur mère suite à un décès ou à une séparation. A priori, les trajectoires susceptibles d'être suivies par ces enfants sont nombreuses, rendant inopérable tout amendement de notre modèle animé par un souci d'exhaustivité. Aussi, contentons-nous ici de nous intéresser aux trajectoires les plus fréquentes et à cet effet d'adopter un petit nombre de règles simples mais suffisamment réalistes de manière à minimiser les modifications à apporter à notre modèle.

Les règles que nous avons adoptées sont les suivantes :

1) Lorsque les enfants en viennent à être séparés de leur mère suite au décès de celle-ci ou bien à une séparation, ils restent en groupe et donc, le cas échéant, sont confiés tous ensemble au même homme, qui est soit le dernier conjoint, si la mère a jusqu'ici contracté au moins une union, soit le père, si elle n'en a jamais contracté.

2) Si les enfants ne passent pas au dernier conjoint ou au père au moment du décès ou de la séparation (pour aller vivre avec des personnes apparentées ou non), ils ne le rejoindront jamais à l'avenir.

Dans ces conditions, le problème à résoudre à propos des trajectoires des enfants séparés de leur mère se réduit au suivi de l'homme ayant hérité de la garde des enfants; toutefois, ce suivi doit débuter non pas quand il en obtient la garde mais bien dès le moment où il entre dans une situation pouvant l'amener à en prendre la garde. Nous devons donc, pour toute femme, suivre en parallèle le statut conjugal de l'homme susceptible d'hériter de la garde des enfants ou en ayant hérité ${ }^{7}$. Il

7 Ce suivi en parallèle de deux personnes est possible parce que le statut conjugal de l'homme à considérer est soit imposé (s'il s'agit du conjoint actuel), soit indépendant de celui de la femme (dans tous les autres cas). 
est possible de réaliser cela 1) en ajoutant aux états originellement considérés un autre état "décédée" et 2) en divisant chacun des états conjugaux au moyen d'un croisement par le statut conjugal de l'ex-conjoint (ou bien du père des enfants) repéré à l'aide d'une échelle de trois valeurs (décédé, seul ou en union).

Un tel élargissement de la variable de statut conjugal requiert évidemment la prise en considération de changements d'état additionnels et donc la disponibilité de données supplémentaires relatives à ces changements. À noter cependant que :

1) certains changements sont régis automatiquement de par les règles adoptées plus haut ${ }^{8}$ et que,

2) au moment de toute rupture d'union, les enfants passent au dernier conjoint ou au père (la parité $\mathrm{p}$ de la mère se voit assigner comme attribut de celui-ci) dans une proportion fixe évidemment moins élevée en cas de séparation qu'en cas de décès (puisque dans le premier cas les enfants restent le plus souvent auprès de leur mère).

La mise en œuvre de notre modèle sur la base d'une définition élargie de la variable de statut conjugal permet en fin de compte d'embrasser les situations où, suite à une rupture d'union, la garde des enfants passe de la mère au dernier conjoint ou au père. En ce cas, le statut conjugal de ce dernier permet aisément de distinguer parmi les situations celles qui correspondent à une famille monoparentale à parent masculin de celles qui correspondent à des familles recomposées.

En fin de parcours, nous nous retrouvons donc à obtenir le nombre et les caractéristiques des familles monoparentales distinguées selon le sexe du parent les dirigeant ainsi que le nombre de familles époux-épouse distinguées en familles recomposées ou non. Reste alors à déterminer la composition de ces dernières, ce qui, compte tenu de ce qu'elle reflète, doit être fait en dehors de notre modèle multidimensionnel. La solution, du moins en ce qui concerne le nombre des enfants dans les familles reconstituées, consiste à faire appel à des tableaux types croisant la parité de la conjointe et la "parité" du conjoint et à modifier ceux-ci de façon à ce que leurs marges s'accordent avec les résultats (nombres d'enfants) déterminés par notre modèle pour chacun des conjoints.

8 Ainsi, par exemple, lorsqu'une femme seule se met en union, l'homme qui lui était associé (ex-conjoint ou père des enfants selon le cas) est immédiatement remplacé par le nouveau conjoint. 


\section{CONCLUSION}

Afin de projeter les familles selon leurs caractéristiques principales, une méthodologie plus appropriée que la traditionnelle méthode des taux de personnes repères car s'appuyant sur les méthodes et modèles de la démographie mathématique multidimensionnelle est en cours d'élaboration. Dans cet article, nous en avons décrit les grandes lignes avec pour principal souci de rendre cette description accessible à tous ceux que ne rebute pas l'utilisation incontournable de la notation matricielle même s'ils ne la maîtrisent que partiellement ou même pas du tout.

La méthodologie en question est ample et complexe; mais son amplitude et sa complexité ne s'opposent en aucune façon à sa mise en application dans une situation concrète. Cependant, faute de disposer de ressources suffisantes, nous ne pouvons mener une telle application que par étapes successives. Au stade actuel de l'avancement des travaux menés de concert avec Heather Juby - laquelle, dans le cadre de sa thèse de doctorat (Juby, 1993), avait entrepris l'opérationnalisation du modèle constituant le cour de la méthodologie initialement proposée (Ledent, Péron et Morissette, 1987) -, nos efforts ont permis de reconstituer de façon très satisfaisante les effectifs de la population féminine du Canada en 1991 en partant des effectifs correspondants observés en 1971.

\section{RÉFÉRENCES BIBLIOGRAPHIgUES}

BONGAARTS, J., 1983. "The Formal Demography of Families and Households: An Overview", Newsletter No. 17. Liège, Belgique, Union internationale pour l'étude scientifique de la population.

BONGAARTS, J., 1987. "The Projection of Family Composition over the Life Course with Family Status Life Tables", dans J. BONGAARTS, T. K. BURCH et K. W. WACHTER, éd., Family Demography: Methods and their Application. Oxford, Angleterre, Oxford University Press : 189-212.

HOEM, J. M., et M. S. FONG, 1976. A Markov Chain Model of Working Life Tables. Université de Copenhague, Danemark, Laboratory of Actuarial Mathematics, WP-2.

JUBY, H., 1993. De la reconstitution à la projection des ménages. Une application au Canada. Montréal, Québec, Université de Montrêal, thèse de doctorat en démographie.

KONO, S., 1987. "The Headship Rate Method for Projecting Households", dans J. BONGAARTS, T. K. BURCH et K. W. WACHTER, 
éd., Family Demography: Methods and their Application. Oxford, Angleterre, Oxford University Press : 287-308.

LAND, K. C., et A. ROGERS, 1982. Multidimensional Mathematical Demography. New York, Academic Press.

LARRIVEE, D., 1990. Projection des ménages et des familles pour le Canada, les provinces et les territoires, 1989-2011. Ottawa, Approvisionnements et Services Canada (pour Statistique Canada). No 91-522 au catalogue (hors série).

LEDENT, J., 1992. Vers des perspectives de familles/ménages sur la base d'un modèle de type multidimensionnel (rapport d'étude remis à Santé et Bien-être national), Montréal, Québec, INRSUrbanisation.

LEDENT, J., 1995. La Projection de la population féminine selon l'état conjugal et certaines caractéristiques des enfants. Texte d'une communication présentée à la Chaire Quetelet 1995, Louvain-la-Neuve, Belgique, 14-16 dẻcembre.

LEDENT, J., Y. PÉRON et A. MORISSETTE, 1987. Ébauche d'une méthodologie nouvelle pour la projection des familles et des ménages au Québec. Québec, Cahier technique du Bureau de la statistique du Québec.

ROGERS, A., 1968. Matrix Analysis of Interregional Population Growth and Distribution. Berkeley, Californie, University of California Press.

ROGERS, A., 1975. Introduction to Multiregional Mathematical Demography. New York, John Wiley and Sons.

ROGERS, A., et J. LEDENT, 1976. "Increment-Decrement Life Tables: A Comment", Demography, 13, $2: 287-290$.

SCHOEN, R., et K. C. LAND, 1979. "A General Algorithm for Estimating a Markovian Generated Increment-Decrement Life Table with Applications to Marital Status Patterns", Journal of the American Statistical Association, 74: 761-776.

USNRC (United States National Resources Planning Committee), 1938. The Problems of a Changing Population. Washington, D. C., U. S. Government Printing Office (cité dans Kono, 1987).

WILLEKENS, F., 1980. "Multistate Analysis: Tables of Working Life", Environment and Planning A, $12: 563-588$.

WILLEKENS, F. J., J. SHAH, J. M. SHAH et P. RAMACHANDRAN, 1982. "Multistate Analysis of Marital Status Life Tables: Theory and Practicen, Population Studies, 36 : 129-144. 


\section{RÉSUMÉ - SUMMARY - RESUMEN}

LEDENT Jacques - VERS UNE PROJECTION DES FAMILLES SELON LEURS CARACTÉRISTIQUES PRINCIPALES

Cet article a pour objet de décrire les grandes lignes d'une méthodologie en cours d'élaboration visant à projeter les familles lou groupes d'individus ayant entre eux des liens conjugaux ou de sang) selon leurs caractéristiques principales, à savoir le genre de famille mais aussi le nombre et l'âge des enfants présents. Cette méthodologie s'appuie pour l'essentiel sur un modèle de type multidimensionnel se présentant comme une extension de la traditionnelle méthode des composantes à la prise en compte du statut conjugal et de la parité des femmes. En bout de course, moyennant un élargissement approprié de la variable de statut conjugal et un prolongement axé sur le cas particulier des familles recomposées, elle autorise un passage immédiat des effectifs de la population féminine selon le statut démographique (issu du croisement selon l'état conjugal et le nombre d'enfants présents) aux effectifs des familles selon leurs caractéristiques principales.

\section{LEDENT Jacques - TOWARDS A PROJECTION OF FAMILIES ACCORDING TO THEIR PRINCIPAL CHARACTERISTICS}

This article describes the main ingredients of a methodology currently under development for projecting families-these groups of individuals linked by conjugal ties or by blood-according to their principal characteristics: family type and number/ages of children. The crux of this methodology is a multidimensional model which can be viewed as an extension of the traditional cohort-components model that incorporates the conjugal and parity statuses of women. With an appropriate generalization of the conjugal status variable and the addition of an appendage for dealing with the special case of step families, this model eventually allows one to shift from the number of women according to demographic status (stemming from cross-classifying conjugal status and numbers of children present) to those of families according to their main characteristics.

\section{LEDENT JaCques - HACIA UNA PROYECCIÓN DE LAS FAMILIAS SEGÚN SUS CARACTERÍSTICAS PRINCIPALES}

En este articulo se describen las principales lineas de una metodología actualmente en curso de elaboración con vistas a proyectar las familias (grupos de individuos relacionados entre sí por vínculos conyugales o de sangre), según sus caracteristicas principales, o sea: tipo de familia, y número y edad de los hijos. Esta metodología se apoya principalmente en un modelo de tipo multidimensional, que puede tomarse como extensión del método tradicional de componentes donde se tiene en cuenta el estatuto conyugal y la paridez de las mujeres. Mediante una ampliación apropiada de la variable de estatuto conyugal, y una prolongación que trate del caso especial de las familias reconstituidas, este modelo permite pasar inmediatamente del número de mujeres según el estatuto demográfico (resultante del cruce del estatuto conyugal y el número de hijos presentes), al número de familias según sus caracteristicas principales. 